\title{
The control of conjunctival fibrosis as a paradigm for the prevention of ocular fibrosis-related blindness. "Fibrosis has many friends"
}

\author{
Peng Tee Khaw $\mathbb{1}^{1,2} \cdot$ Yann Bouremel ${ }^{1,2,3} \cdot$ Stephen Brocchini ${ }^{1,2,3} \cdot$ Christin Henein $\mathbb{1}^{1,2,3}$
}

Received: 15 February 2020 / Revised: 19 May 2020 / Accepted: 9 June 2020 / Published online: 25 June 2020

(C) The Author(s), under exclusive licence to The Royal College of Ophthalmologists 2020

\begin{abstract}
The processes involved in ocular fibrosis after disease or ocular tissue injury, including surgery play an important part in the development or failure of treatment of most blinding diseases. Ocular fibrosis is one of the biggest areas of unmet need in ophthalmology. Effective anti-scarring therapies could potentially revolutionise the management of many diseases like glaucoma worldwide. The response of a quiescent or activated conjunctiva to glaucoma surgery and aqueous flow with different stimulatory components and the response to different interventions and future therapeutics is a paradigm for scarring prevention in other parts of the eye and orbit. Evolution in our understanding of molecular and cellular mechanisms in ocular fibrosis is leading to the introduction of new and re-purposed therapeutic agents, targeting a wide range of key processes. This review provides current and futures perspectives on different approaches to conjunctival fibrosis following glaucoma surgery and highlights the challenges faced in implementing these therapies with maximal effect and minimal side effects.
\end{abstract}

\section{Introduction}

Ocular fibrosis after disease or injury, including surgery, is a complex biological process and features in many blinding ocular diseases, which result from autoimmunity (e.g. pemphigoid and fibrosis in dysthyroid eye disease), angiogenesis and wound healing (e.g. glaucoma surgery and capsular opacification). Fibrovascular scarring features in numerous ocular conditions such as, diabetic retinopathy, macular degeneration, proliferative vitreoretinopathy, retinopathy of prematurity, and neovascular glaucoma, and is a consequence of the underlying inflammatory or hypoxiadriven neovascularization. Therefore, prevention of underlying drivers is the most appropriate therapeutic approach to preserve visual function. Furthermore, scarring is a major

Peng Tee Khaw

p.khaw@ucl.ac.uk

1 National Institute of Health Research Biomedical Research Centre at Moorfields Eye Hospital and UCL Institute of Ophthalmology, London, UK

2 UCL Institute of Ophthalmology, London, UK

3 UCL School of Pharmacy, London, UK potential obstacle to regenerative therapies such as cellular transplantation [1].

Collectively, ocular fibrosis results in a huge amount of ocular morbidity worldwide. Many of these conditions were discussed during the XLIX Cambridge Ophthalmological Symposium, which was chaired by Professor Sir Peng Tee Khaw. Discussion during the symposium drew on lessons learnt from aberrant wound healing pathogenesis and strategies to lessen scarring after GFS and ocular fibrosis in general. Improved understanding of the molecular and cellular mechanisms of wound healing processes, and the mode and mechanism of action of modulating agents, has led to the identification and modulation of potential therapeutic targets, and more effective use of existing agents. This paper aims to provide an overview of some of the approaches used to modulate conjunctival fibrosis after GFS and how these approaches can be applied to other forms of ocular fibrosis.

In glaucoma surgery the intent is to make a drainage fistula from the anterior chamber to the sub-Tenon space to allow aqueous outflow and lower the intraocular pressure (IOP). In this paper we will concentrate on glaucoma surgery involving fistulas draining externally to the sub-Tenon space. The healing response and the fibrosis that occurs are the primary determinants of the final IOP. This is because conjunctival fibrosis determines the hydraulic conductivity 
of the conjunctiva, which controls the fluid flow through the tissue. IOPs between 10 and $14 \mathrm{mmHg}$ are associated with minimal fibrosis of the conjunctiva, and slower rates of visual field deterioration [2].

Several different stimulatory components arise from glaucoma surgery, which activate quiescent conjunctiva. Conjunctival fibrosis can result in the failure of conventional bleb-forming glaucoma filtration surgeries. New drainage implants employing different outflow pathways including subconjunctival, trabecular meshwork/Schlemm's canal and suprachoroidal routes of drainage are used clinically. All of these drainage implants are eventually limited by fibrotic reaction around the device regardless of its mode of action. Despite advances in glaucoma surgical techniques, devices and post-operative care, fibrosis remains the principal impediment to long-term control of IOP following surgery.

\section{Conjunctival wound healing responses after glaucoma surgery}

Like all wound healing, conjunctival wound healing involves a complex interplay between growth factors, cytokines, receptors and enzymes and cross-talk between different cell types. The early phase of conjunctival wound healing following glaucoma surgery involves leakage of plasma proteins (fibrinogen, plasminogen and fibronectin) and blood cells (platelets, polymorphonuclear neutrophils and red blood cells) from disrupted vasculature. Conversion of fibrinogen to fibrin results in fibrin clot formation and blood coagulation. The combination of fibrin clot formation and released proteins, lead to migration and attraction of neutrophils, macrophages, and lymphocytes to the surgical site during the inflammatory phase [3].

Pleiotropic genes, involved in conjunctival fibrosis include, but not limited to, transforming growth factor-B (TGF-ß), vascular endothelial growth factor (VEGF), platelet-derived growth factor (PDGF), fibroblast growth factor (FGF) and connective tissue growth factor (CTGF), which contribute to wound healing regulation. Aqueous contains a number of growth factors, including transforming growth factor (TGF- $\beta$ ), which is a main mediator of fibrosis during wound healing $[4,5]$. The later phases of conjunctival fibrosis can be subdivided into inflammation, proliferation, and tissue remodelling (see Fig. 1). During the proliferation phase, activated fibroblasts and endothelial cells migrate to the site of injury, and granulation tissue formation and angiogenesis ensues. Activated Tenon fibroblasts proliferate and lay down extracellular matrix (ECM). During tissue remodelling, blood vessels regress and fibroblasts adopt a myofibroblast phenotype which induces cross-linking of collagen type 1 and elastin resulting in scar formation.
The increase in the number of fibroblasts during the proliferative phase, starts as early as two days post-operatively, and provides an initial advantage to maintain the integrity of the conjunctiva and protect the eye against infection. This reflects an evolutionary pressure towards tissue fibrosis in response to injury. The degree of tissue damage and the host response in terms of severity and duration of inflammation, determines the extent of conjunctival scarring. Moorfields Safer Surgery System, is a modified surgical technique, which aims to minimise the insult and modulates the effects of antimetabolites [6]. The extent of conjunctival fibrosis after glaucoma filtration surgery (GFS) will vary between individuals, as it is influenced by risk factors such as, pre-existing inflammation, duration and type of eye drop therapy and a previous history of surgery [7]. Other drivers of fibrosis after GFS include suture material, aqueous humour derived growth factors, mechanical transduction from aqueous fluid flow, signalling molecules released from ECM storage sites, myofibroblast trans-differentiation leading to further matrix deposition [8]. Post trabeculectomy, the Moorfields bleb grading system [9] is a useful tool to predict long-term IOP outcome. The grading system assesses inflammation and effective application of antimetabolites [10].

\section{The fibroblast: origins, activation, differentiation and proliferation}

Following GFS, fibrosis is mediated primarily by Tenon fibroblasts and their precursors, myofibroblasts. Myofibroblasts are the key effector cells resulting in surgical failure. Myofibroblasts are derived from either one or more origins irrespective of the nature of the initial injury. The exact origin of myofibroblasts during conjunctival fibrosis is debated, and beyond the scope of this paper. Nevertheless the diversity of myofibroblast progenitor cells is relevant in the development of novel therapies. In brief, myofibroblasts involved in conjunctival fibrosis might originate from beyond locally residing cells in the sclera, conjunctiva and Tenons, such as circulating bonemarrow derived- fibroblasts (see Fig. 2). The relative contribution of the different origins of progenitor conjunctival myofibroblasts is unknown due to the lack of a specific myofibroblast cell marker. Myofibroblasts present microfilament bundles expressing $\alpha$-smooth muscle actin ( $\alpha$-SMA), responsible for wound construction. Myofibroblasts deposit an abundant amount of ECM, produce crosslinking enzymes, and inhibitors of ECM, degrading metalloproteinases. Persistent activation of myofibroblasts and mechanical stress such as that found in a tight encapsulated bleb potentiates profibrotic factors. Myofibroblast contractility attracts macrophages, which modulate ECM composition and stiffening [11]. 
Fig. 1 Sequence of events after glaucoma surgery. Patient risk factors should be considered and mitigated against to modulate conjunctival fibrosis after surgery (a). Surgical creation of the aqueous outflow pathway and suture material results in the release of blood constituents and release of cytokines and growth factors from the aqueous, Tenons and sclera (b). In addition to these factors, fibrin clot initiates infiltration of macrophages and polymorphic neutrophils to the bleb site (d). Activation, elongation, and proliferation of Tenon fibroblasts ensues with significant deposition of extracellular matrix (ECM), which persists due to stimulatory tractional forces and growth factors (e). Apoptosis of fibroblasts and vascular endothelial cells block outflow of the aqueous humour resulting in surgical failure due to scarring (f). (Adapted with permission).
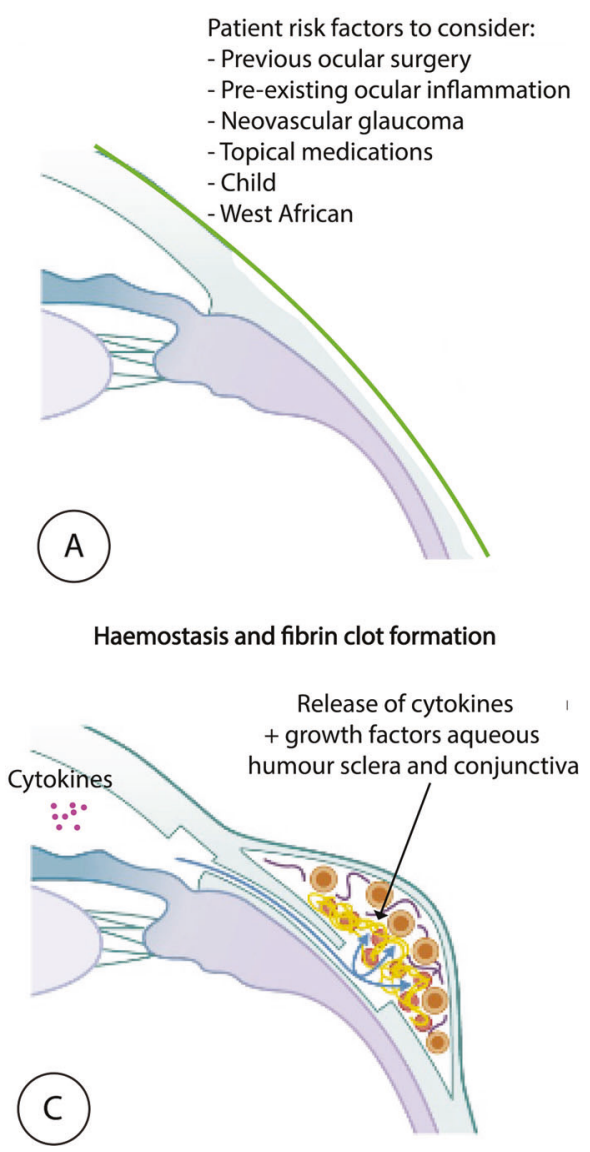

Proliferation and tissue remodelling

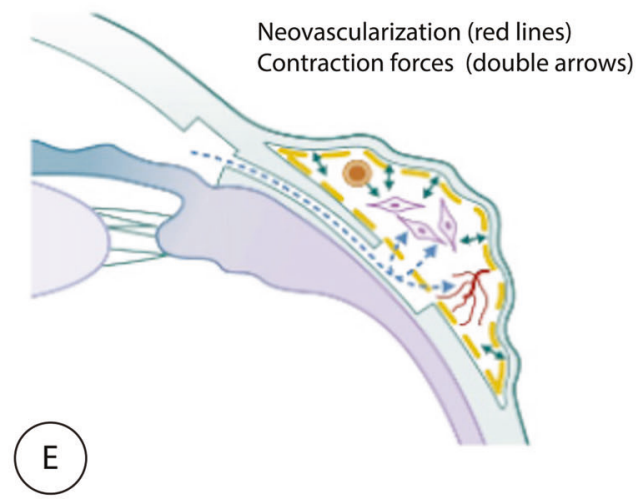

Glaucoma filtration surgery: Creation of the aqueous outflow pathway

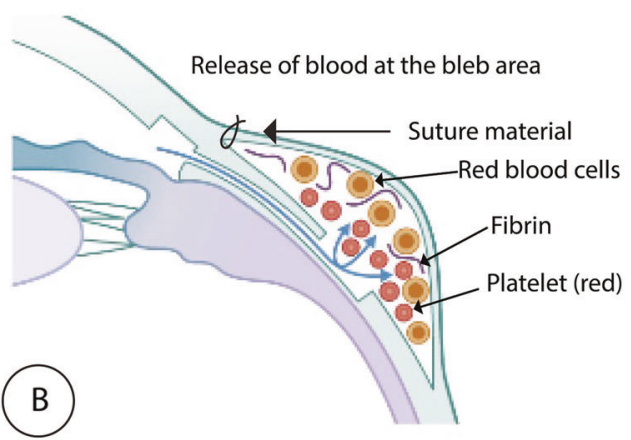

Inflammation: Infiltration of macrophages and PMNs at the bleb area
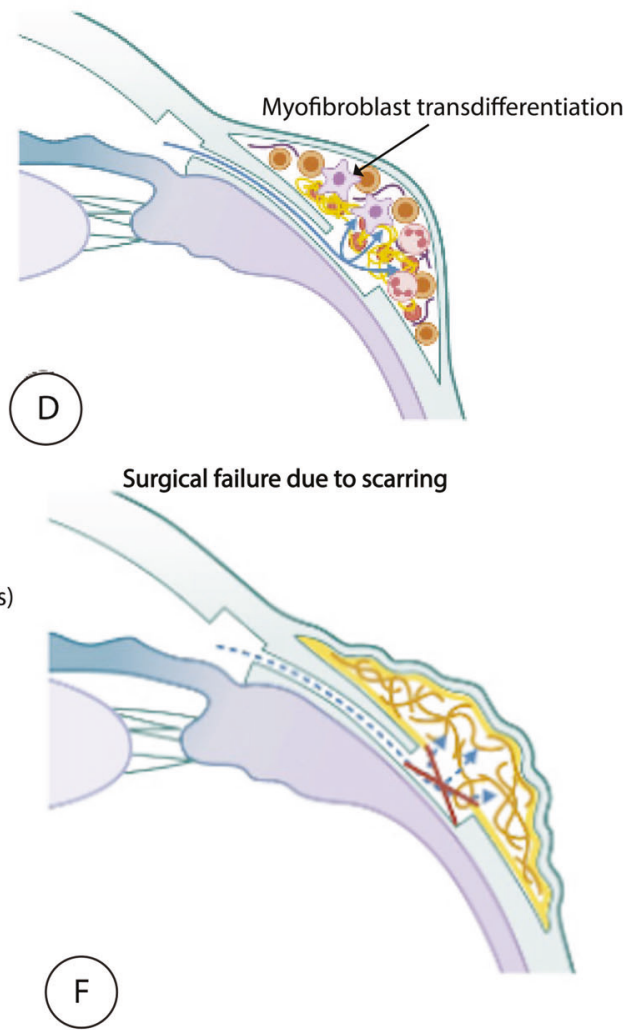

\section{Therapies targeting fibrin clot}

Fibrin clot formation is an important step of the wound healing process. Fibrinolytic agents, such as tissue plasminogen activator, were thought to be useful to dissolve fibrin and blood clots after glaucoma surgery. Urokinase is a thrombolytic agent that could be used as an alternative to tissue plasminogen activator, and intracameral urokinase injection reduced IOP after glaucoma surgery in the short term [12]. Prolonged bleeding is the main side effect that deters the use of these agents, and in addition fibrin breakdown products can stimulate the proliferation of fibroblasts. Treatment with saratin, an anti-thrombotic and anti-fibrotic agent, in a rabbit GFS model, improved bleb survival when administered as two postoperative subconjunctival injections when compared with topical saratin treatment alone [13].

\section{Therapies targeting inflammation}

Reducing inflammation is a standard paradigm for preventing scarring after surgery or injury as chronic 


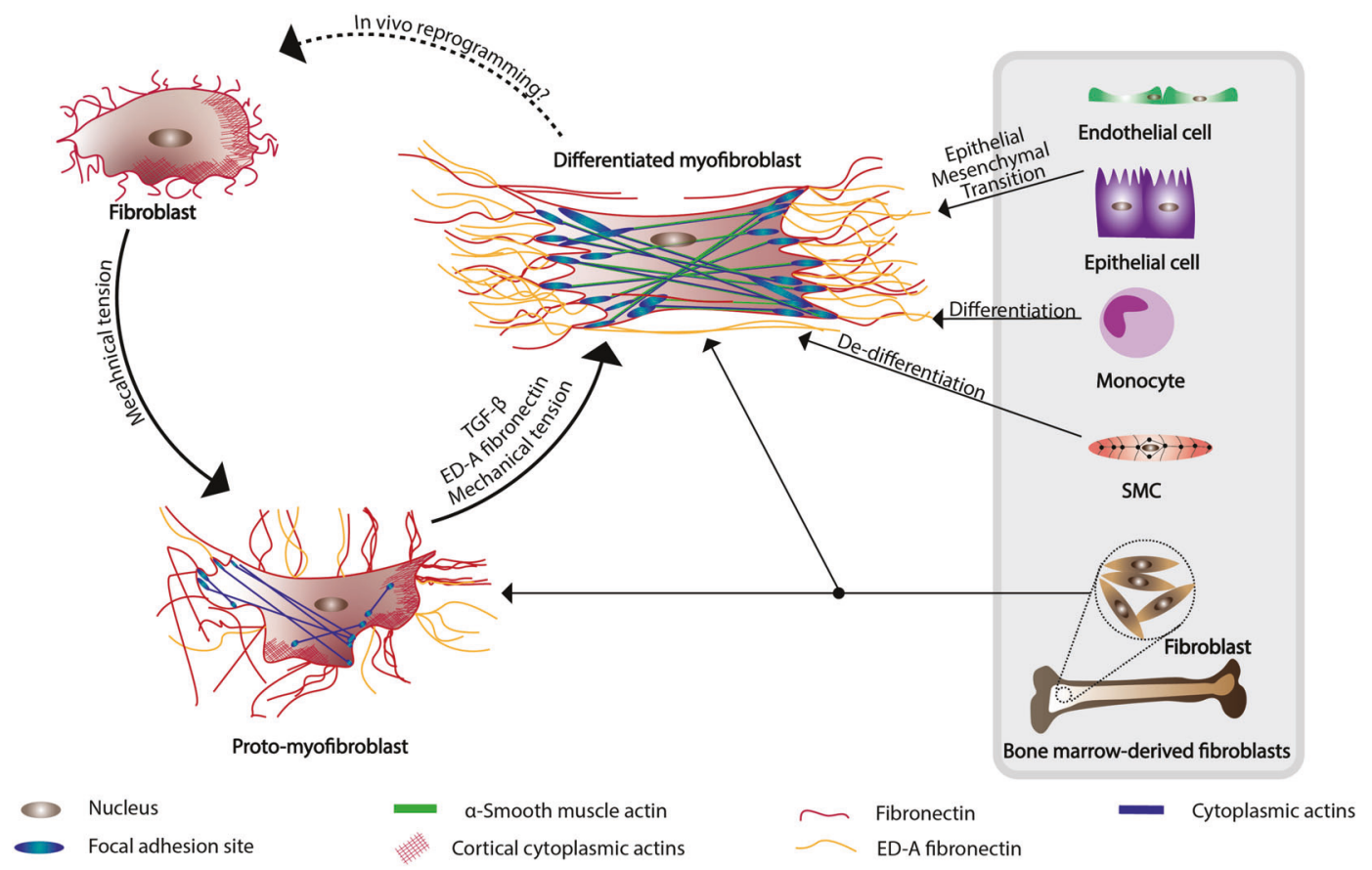

Fig. 2 Origins of myofibroblast precursors and stages of myofibroblast differentiation. Myofibroblasts precursors may originate from locally residing cells in the sclera, conjuntiva and Tenons layer or from circulating bonemarrow derived fibroblasts. SMC smooth muscle cell, $T G F-\beta$ transforming growth factor-beta.

inflammation is associated with much more fibrosis. Broadspectrum anti-inflammatory drugs are standard therapy post-operatively, and are usually administered as topical steroids. The use and tapering of topical steroids such as prednisolone acetate $1 \%$ are an important feature of the post-operative care to modulate inflammation and maintain adequate intraocular pressure control. Steroids can be started prior to surgery if the eye is inflamed. The use of topical non-steroidal anti-inflammatory agents (NSAIDs) after glaucoma surgery remains controversial, as these agents may be associated with a greater incidence of wound problems such as bleb leakage [14]. Nonetheless, where inflammation persists despite steroid use, NSAIDs may be a useful adjunct.

Studies have investigated others methods of administration to enhance steroid bioavailability and effectiveness in dampening the inflammatory response. A randomised controlled trial showed intra-operative sub-Tenon triamcinolone during augmented trabeculectomy surgery in patients with secondary glaucomas did not improve success rate or reduce complications [15]. In another study with a small sample size, intracameral triamcinolone showed no benefit after heterogenous glaucoma operations with or without use of mitomycin-C (MMC) [16].

Synthetically engineered macromolecules such as dendrimer glucosamine and cyclodextrins have been developed to enhance steroid delivery in experimental studies. Encapsulation of drugs in colloidal carriers liposomes and nanoparticles can enhance permeation across cell membranes and prevent enzymatic degradation [17]. Also, ocular implants are being investigated as alternatives to topical or injectable steroid drug delivery systems. Subconjunctival sustained-release dexamethasone (Ozurdex) have been piloted as an adjunct to trabeculectomy [18]. Ozurdex is comprised of a biodegradable co-polymer of lactic acid and glycolic acid so does not require surgical removal.

Anti-inflammatory drug loaded contact lenses have been developed to increase residence time of steroids in the tear film. Contact lenses fabricated from poly-2-hydroxyethyl methacrylate hydrogels with drug molecules entrapped into a nanoparticulate system can provide controlled drug release over days [19]. Due to increased residence time, drug permeation through the tissue can offer prolonged exposure. The delivery rate can be further modified by altering the loading dose in the hydrogel. However, this drug delivery system has yet to be tested in the context of GFS post-operative care.

One would assume favourable outcomes by targetting 'upstream' immune responses. However, broad-acting immunosuppressive therapies have proved sub-optimal to date, and have not entered routine use for glaucoma surgery. Calcineurin inhibitors such as cyclosporine, tacrolimus and sirolimus, are immunosuppressive agents that reduce the production of interleukin-2 (IL2) and IL2 receptor expression. Following GFS, topical cyclosporine, had no effect on IOP, although ocular surface disease improved [20]. A 
combination of therapies targeting several different pathways or the development of novel agents addressing multiple components of fibrosis following glaucoma surgery appear to be required.

\section{Therapies targeting proliferation}

Antimetabolites, 5-fluorouracil (5-FU) and MMC are conventionally used at the surgical site to limit conjunctival scarring following GFS. While these agents have been shown to improve clinical outcomes, they do so in a nonselective manner inhibiting the proliferation of subconjunctival Tenons fibroblasts. Consequently, use of antimetabolites is associated with a significant side-effect profile that can lead to hypotony, bleb leakage, blebitis, endophthalmitis and vision loss.

5-FU is an effective inhibitor of Tenons fibroblast growth by antagonising pyrimidine metabolism inhibiting DNA synthesis and ultimately resulting in cell death [21]. Use of 5-FU at high concentrations, however, often leads to corneal epithelial toxicity and apoptosis, since 5-FU is toxic to all actively replicating cells. Intraoperative or post-operative 5-FU significantly reduces the risk of trabeculectomy failure but causes increased rates of wound leakage, hypotony and epitheliopathy compared with trabeculectomy alone [22-24].

MMC exhibits antiproliferative effects by acting as a DNA cross-linking alkylating agent that can interfere with cells at any stage of the cell cycle, inhibiting DNA replication, cell mitosis and protein synthesis. MMC increases the success rate of GFS, mainly through inhibition of proliferation of fibroblasts. MMC is more potent than 5-FU, inhibiting proliferation for a longer period [25, 26]. Metaanalysis of 11 studies provided some evidence that favoured MMC to 5-FU in lowering IOP at 1-year post trabeculectomy. Less epitheliopathy and hyphaema was observed with MMC [27]. The method of applying the MMC is also critical and has a huge bearing on the long-term bleb appearance and complications, and these general physical principles of drug applications are also very important in the prevention of scarring [28].

Beta-radiation only has superficial tissue penetration and can be used over the conjunctival bleb to arrest cell growth rather than cause widespread cell death. Tenons fibroblasts exposed to beta-radiation express high levels of p53 to prevent cell replication in the presence of DNA damage, decreasing ECM production [29]. $\beta$-radiation can be applied locally, very rapidly (in $<1 \mathrm{~min}$ ) and accurately, making it a useful anti-fibrotic option in GFS. The current 1000cGy $\beta$ radiation dose is based on cell culture studies [30] and is much lower compared with dosages historically used to treat pterygium, which varied from 2000 to $6000 \mathrm{cGy}$. The protocols to apply these higher doses of $\beta$-radiation had a poor safety profile in pterygium management [31]. In GFS $\beta$-radiation has proven to be an effective anti-fibrotic therapy in high-risk patient groups such as patients from West African descent. A meta-analysis including four studies showed intraoperative beta irradiation lowered the risk of surgical failure compared with trabeculectomy alone [32]. However, the $\beta$-radiation group had a higher risk of cataract [32], but this may have been associated with lower pressures which is less often seen with newer GFS such as the Moorfields Safer Surgery System [6]. When $\beta$-radiation was compared with intraoperative 5-FU, there was no difference in risk of failure or complication following phacotrabeculectomy [33]. More recently, randomised trials have shown that $\beta$-radiation with MMC are superior to MMC alone [34]. In a South African study, eyes receiving $\beta$ radiation were 5.55 times more likely to have an IOP $<16$ $\mathrm{mmHg}$ on no medical treatment compared with eyes receiving MMC [35]. New and improved radiation delivery systems and sources should allow treatment times as short as 30 seconds.

The use of an adenoviral delivery system may also be considered feasible. The $p 21$ gene is an important downstream effector of the $p 53$ gene, which is a tumour suppressor gene that arrests cell cycle progression. A recombinant adenovirus carrying human $p 21$ gene inhibited the proliferation of Tenon's capsule fibroblasts, prevented conjunctival scarring, and significantly reduced IOP in animal models of GFS [36].

\section{Therapies targeting growth factors}

Stimulatory growth factors and cytokines, released at the site of surgery can both amplify and reduce inflammation during the wound healing process (Fig. 3). Identifying therapeutic targets can be challenging due to the pluripotency and redundancy of these different signalling molecules [37]. TGF- $\beta$ is instrumental in numerous processes of fibrosis, such as stimulating inflammation, migration of cells, loss of cell-cell adhesions, epithelialmesenchymal transition into myofibroblast phenotype and modulates ECM deposition.

\section{Transforming growth factor- $ß$}

TGF- $\beta 2$ is the predominant intraocular TGF- $\beta$ isoform associated with ocular fibrosis such as proliferative vitreoretinopathy, posterior capsular opacification and fibrosis after GFS [38-41]. Lerdelimumab, a monoclonal antibody to TGF- $\beta 2$ was trialled to prevent the progression of fibrosis in primary trabeculectomy. The dose level and treatment regimen were derived from rabbit studies [42]. 


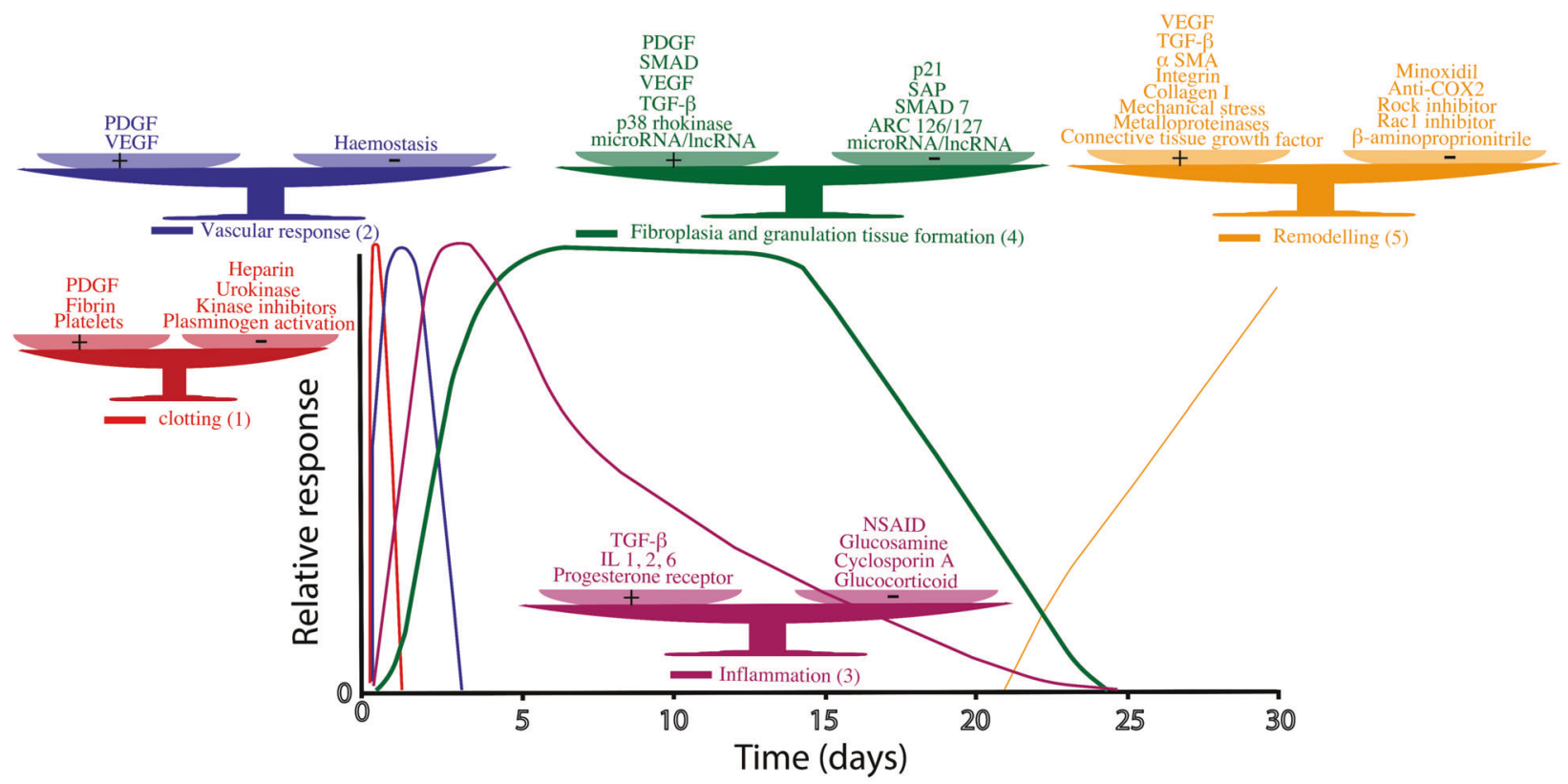

Fig. 3 Phases of conjunctival wound healing. Phases include clotting, vascular response, inflammation, fibroplasia and tissue remodelling/scarring [37]. Conjunctival wound healing involves a complex interplay between growth factors, cytokines, receptors and enzymes, and cross-talk between different cell types. Some of the mediators and

There was no difference between Lerdelimumab and placebo in preventing surgical failure in a phase III human trial [43]. The use of a monoclonal antibody directed solely against TGF- $\beta 2$ may represent too narrow of an approach since other isoforms of TGF- $\beta$ are also involved. The very short subconjunctival drug half-life and the mode of delivery may not have been optimal to neutralise TGF- $\beta 2$, and the absence of neutralisation of the other isoforms may have also been a disadvantage. A pan-TGF- $\beta$ antibody, Fresolimumab, which neutralises all three main isoforms of TGF- $\beta$, may have potential applications in the treatment of a broad range of medical conditions [44]. To our knowledge Fresolimumab has not been evaluated in the context of ocular fibrosis and may not be appropriate in the context of corneal-related fibrosis which requires a more targeted approach to downstream TGF- $\beta$ signalling pathway mediators, in order to maintain normal corneal wound healing and corneal transparency [45].

RNA sequencing analyses have identified long noncoding RNAs (LncRNAs) and short RNA molecules (miRNAs) involved in conjunctival fibrosis, as new approaches to targeting the TGF- $\beta$ signalling pathway. MicroRNAs are non-coding molecules and function to silence gene expression or targeted degradation of messenger RNAs. MicroRNAs regulate many biological processes, including tissue fibrosis and their function is likely to be tissue and species specific [46, 47]. A study performed to identify miRNAs that inhibit the transcription of factors involved in each phase are listed in the balances. PDGF platelet-derived growth factor, $V E G F$ vascular endothelial growth factor, $T G F-\beta$ transforming growth factor-beta, $S A P$ serum amyloid $\mathrm{P}$ and anti-COX2 anti-cyclooxygenase 2 .

profibrotic proteins in Tenon fibroblasts showed that overexpression of miRNA-29b, targets profibrotic TGF- $\beta$ signalling pathway, inhibited production of collagen type I- $\alpha 1$ and protected the conjunctiva from fibrosis [48]. Data from other specialities suggest, inhibition of miRNA-21 and overexpression of miRNA-29 seem to be promising antifibrotic approaches $[49,50]$. Potential drugs in the development pipeline, which could be repurposed include an inhibitor of miRNA-21, RG-012 and is under evaluation for the treatment of Alport syndrome. There is also a miRNA29 mimic, MRG-201, that is being assessed to treat cutaneous scleroderma [51].

LncRNAs do not code for proteins instead they interact with chromatin to modify gene expression. LncRNAs induced by TGF- $\beta / \mathrm{Smad} 3$ signalling have been identified in human tenon fibroblasts obtained from patients who had undergone GFS [52]. Although evidence is limited, IncRNAs are likely to have a function in conjunctival fibrosis and could present new areas for future investigation [53-55].

\section{Vascular endothelial growth factor}

Angiogenesis is a crucial process in wound closure by enabling inflammatory cells and fibroblasts to migrate to wound site, and is mediated by VEGF $[56,57]$. VEGF is one of the most potent vasodilators known and may facilitate the extravasation of blood proteins and cytokines. 
VEGF among other growth factors is found to be upregulated in the aqueous following GFS [58]. There remains uncertainty whether anti-VEGF agents such as bevacizumab and ranibizumab are as effective in preventing fibrosis following trabeculectomy as MMC or sham control [59]. The optimal dose and frequency of applications of anti-VEGF in the context of GFS remains unclear. Better bleb morphology and reduction in bleb vascularisation has been observed following intravitreal ranibizumab in combination with intraoperative topical MMC [60]. Subconjunctival bevacizumab has been used successfully to rescue failing filtering blebs that exhibit neovascularization [60, 61]. AntiVEGF treatments have a role in the treatment of neovascular glaucoma, a disease where angiogenesis is the underlying pathology $[62,63]$.

\section{Platelet-derived growth factor}

Platelet-derived growth factor-B (PDGF-B) is another growth factor that can activate certain ocular fibroblasts. Aptamers are nucleic acid-based macromolecules that can recognise, bind, and block PDGF-B with high affinity and specificity. Two aptamers, ARC126 and ARC127, have been tested in a rabbit model of proliferative vitreoretinopathy [64], and might also be useful in glaucoma surgery.

There are also potential kinase inhibitors that can be used for anti-fibrotic therapy. Nintedanib inhibits PDGF receptor, VEGF receptor and FGF receptor tyrosine kinases. Nintedanib is FDA approved as an orally administered medicine to treat idiopathic pulmonary fibrosis (IPF). An in vitro study using human Tenons fibroblasts showed Nintedanib inhibits proliferation and motility induced by TGF- $\beta 1$ [65]. Pirfenidone is also an orally administered medicine used to treat IPF. While the mode of action of Pirfenidone has not been firmly established, it appears there is reduced fibroblast proliferation and collagen production [66]. Both Nintedanib and Pirfenidone may be two of the few drugs licenced for fibrosis because of the multiple possible mechanisms of action given the many cross-overs covering biological systems responsible for wound healing and fibrosis.

\section{Tissue remodelling - therapies targeting matrix, collagen and enzymes}

Mechanical stress induces the protomyofibroblast phenotype and expression TGF- $\beta$, which is critical for the final myofibroblast transformation. In addition to mechanical stress and TGF- $\beta$, integrin signalling are also necessary for the maintenance of the myofibroblastic phenotype [67]. TGF- $\beta$ upregulates CTGF expression, which is another important fibrogenic cytokine that facilitates a persistent pro-fibrotic state. Drug targeting either CTGF or TGF- $\beta$ signalling can maintain filtering bleb function by minimising scarring [68-70]. Blocking of CTGF using an antibody against TGF- $\beta 2$ receptors reduced conjunctival scarring in ocular cicatricial pemphigoid [71].

The expression of matrix metalloproteinases (MMPs) varies throughout wound healing as they contribute to ECM remodelling by degrading ECM proteins. MMPs mediate inflammation by stimulating fibroblasts, altering endothelial permeability, and activating VEGF [56, 72, 73], and are known to be upregulated in the fibroblasts of the bleb walls surrounding glaucoma implants [74]. Ilomastat, a broadspectrum MMP inhibitor, can reduce subconjunctival scarring after experimental GFS [75]. A cyclodextrin-Ilomastatcomplex suspension eye drop could permeate through conjunctival tissue and therapeutic concentrations were present in the anterior chamber, conjunctiva and sclera in a preclinical in vivo study [76]. Doxycycline was found to inhibit MMPs but to a lesser extent than MMC in a rabbit model [77]. Sustained drug delivery is crucial to maintain beneficial drug effect throughout the ECM remodelling phase of wound healing.

Other enzymes such as lysyl oxidase (LOX) and lysyl oxidase-like (LOXL), crosslink collagen and elastin in the ECM, which leads to fibrosis [78]. A monoclonal antibody to LOXL-2 reduced both inflammation as well as fibrosis in vivo [79]. Elevated TGF and LOXL-2 levels in Tenon's tissue at the time of GFS strongly correlated with surgical failure at 1-year, suggesting LOX-2, could be a promising therapeutic target for reducing scar formation after glaucoma surgery $[79,80]$.

Rho and Rac1 are small GTPases that play a critical role in the regulation of actomyosin cytoskeletal organisation and cell motility. A Rac1 inhibitor, efficiently inhibited fibroblast-mediated matrix contraction, reduced MMP-1 expression in conjunctival tissue and did not cause any significant toxicity [81]. The Rho-associated protein kinase (ROCK) inhibitor, also reduced fibroblast-mediated matrix contraction in vitro, and improved bleb survival in experimental GFS [82].

Activated myofibroblasts are the key effector cell in conjunctival fibrosis and scar formation as previously described. Myocardin-related transcription factor/serum response factor (MRTF/SRF) transcription pathway plays an important role in myofibroblast activation in fibrosis and is a key upstream regulator of MMP expression in ocular fibrosis. MRTF inhibition can be achieved through siRNAmediated silencing or pharmacological inhibition. A few small molecule inhibitors, have been reported to be more effective in reducing MRTF/SRF-regulated gene transcriptional signalling than ROCK inhibitors [83]. Sustained release liposome MRTF/SRF pathway inhibitors have shown efficacy in preventing conjunctival fibrosis in an established rabbit model of GFS [84, 85]. 


\section{Delivery of anti-fibrotic therapies}

Application of anti-fibrotic agents at the bleb site is hampered by rapid clearance from the subconjunctival space due to aqueous outflow resulting in conjunctival absorption and systemic clearance. Mediating the localised healing response after surgery for extended periods of time may best be achieved by prolonging the presence of a widespectrum anti-fibrotic agent in the subconjunctival space. Developing long acting dosage forms for subconjunctival administration require that material-tissue interactions are optimised to avoid additional localised inflammation due to foreign body responses. Sustained release implants and in situ forming gels have been described for intraocular and subconjunctival use, which may potentially be developed for administration after GFS or drainage device implantation. Strategies for the administration of anti-fibrotic therapies including sponges, hydrogels, injections, implants, gene therapies and cell-based therapies are shown in Fig. 4 and Table 1.

Local accumulation in the sub-tenon space can be achieved with the use of particulate associated formulations (i.e. nano or micro sized colloids). Particulate carriers have been described that bind to cell-surface receptors on myofibroblasts and facilitate drug internalisation, which is triggered by binding to the receptor. Theoretically such strategies may increase focal conjunctival anti-fibrotic activity with lowered unwanted side effects and toxicity to other ocular tissues. Following glaucoma surgery, there is overexpression of LDL receptor in activated Tenons fibroblasts [86]. Engineered LDL-MMC-nanoparticles may provide a novel target for drug delivery systems that specifically bind to LDL receptors mainly in activated fibroblasts. This may achieve highly selective targeting, smaller drug dose requirement, better bioavailability and reduced cellular toxicity to quiescent cells [86].

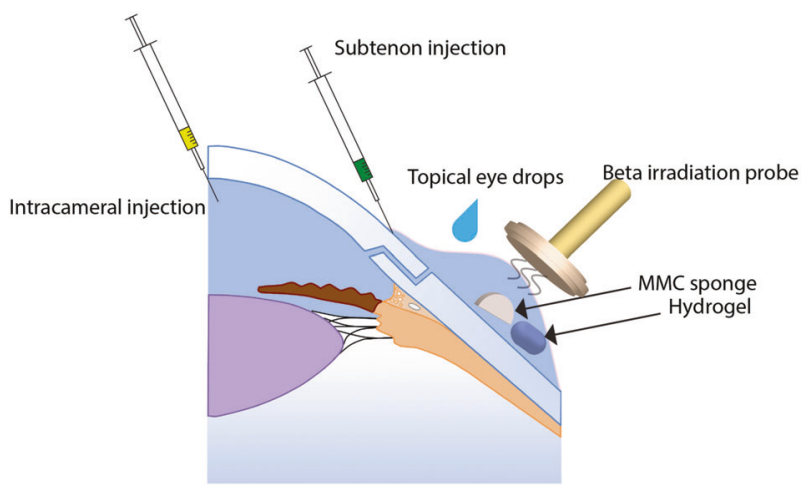

Fig. 4 Delivery of anti-fibrotic therapies. Localised treatment options are presented, some are based on the development of subconjunctival specific dosage forms that can prolong the local exposure of an anti-fibrotic agent at the bleb site.

\section{Surgical principals and modifications}

Simple changes in surgical techniques and the application of antimetabolite treatment have significantly improved the success rate and reduced the complication rate of glaucoma surgery [6] such as minimising tissue damage and application of tailored dose of anti-fibrotic agent. The principle of using physical spacers to attenuate mechanical stress might also help to maintain the subconjunctival pocket after GFS. Spacers using human amniotic membrane, perfluoropropane gas, or sodium hyaluronate, have been reported to increase bleb survival and to lower IOP [87]. More research is needed to develop better biocompatible materials that would lead to less scarring and better postoperative results after glaucoma surgery.

Use of ocular anti-hypertensives after surgery may play a role in conjunctival fibrosis and tissue remodelling. After glaucoma drainage device surgery, the use of aqueous suppressants is preferable to prostaglandin analogues to treat raised IOP [88, 89]. In experimental models prostaglandin analogues induced collagen contraction [90], upregulated interleukin-2 and MMP-9, while aqueous suppressants decreased expression of $\alpha$-SMA, reducing the transformation of fibroblasts to myofibroblasts [91]. Aqueous suppressants may attenuate growth factors derived from the aqueous and reduce the mechanical stress from aqueous pressure in the bleb.

Needling with 5-FU or MMC augmentation, can be performed to rescue failing blebs particularly in high risk cases. Bleb failure can occur early or late following GFS. Fibrosis can occur at the level of the conjunctival, episcleral or internal ostium. Needling was most successful soon after trabeculectomy, but rescue of late failed trabeculectomy bleb has a lower likelihood of success [92]. Therapies targeting early tissue remodelling phases may influence longterm post-operative outcomes, as it is the overall accumulation of fibrotic tissue that leads to pressure rise and ultimate surgical failure.

Concomitant surgery may worsen fibrosis such as the combination of phacoemulsification with glaucoma drainage surgery. A greater amount of post-operative inflammation and flare have been observed after cataract surgery compared with trabeculectomy for up to 6 months surgery. Inflammation associated with cataract surgery is likely to be a result of crystallin lens protein, ultrasound energy and the speed and volume of intraocular fluid irrigation during surgery. The consequence is an upregulation of pro-fibrotic cytokines and growth factors in the aqueous humour and increased risk of bleb failure. The extent of time these profibrotic proteins are upregulated should be borne in mind when prescribing postoperative anti-inflammatory agents in cases of phacotrabeculectomy and sequential surgeries, (cataract surgery after trabeculectomy), if excessive scarring is to be reduced [93]. 
Table 1 Currently available treatment modalities to modulate wound healing during and after glaucoma surgery.

\begin{tabular}{lll}
\hline Therapeutic agent & Mode of delivery & Effect \\
\hline Mitomycin-C, 5-Fluorouracil & $\begin{array}{l}\text { Sponge } \\
\text { Subconjunctival injection }\end{array}$ & Decrease fibroblast proliferation, apoptosis \\
Beta-radiation & $\begin{array}{l}\text { Local irradiation of } \\
\text { tissues }\end{array}$ & Decrease fibroblast proliferation \\
Local anaesthetic & $\begin{array}{l}\text { Sub-Tenon's injection } \\
\text { Subconjunctival injection }\end{array}$ & $\begin{array}{l}\text { Might decrease pain and local } \\
\text { inflammation }\end{array}$ \\
Steroid & $\begin{array}{l}\text { Topical } \\
\text { Ozurdex implant }\end{array}$ & $\begin{array}{l}\text { Decrease IOP } \\
\text { Prolong bleb survival }\end{array}$ \\
Nonsteroidal anti-inflammatory & Topical & $\begin{array}{l}\text { Decrease IOP but greater incidence of } \\
\text { wound leaks and conjunctival retraction }\end{array}$ \\
drug (NSAID) & Subconjunctival injection & $\begin{array}{l}\text { Controversial: some studies report } \\
\text { improved bleb survival and lower IOP. } \\
\text { Anti-VEGF (Bevacizumab) }\end{array}$ \\
& Intracameral injection & $\begin{array}{l}\text { Decrease needling interventions after } \\
\text { trabeculectomy }\end{array}$ \\
\hline
\end{tabular}

The earlier cataract surgery is performed within 6 months, the greater the risk of bleb failure [94].

\section{Can fibrosis be reversed?}

Direct in vivo reprogramming of myofibroblasts into fibroblasts has potential as a strategy for regeneration in fibrosisrelated conditions. Hepatic fibrosis has been reversed by using transcription factor induction, directly inducing myofibroblasts to transform into hepatocytes using a lentiviral vector in mouse models of chronic liver disease [95]. For this regenerative activity to be achieved in glaucoma surgery, transcriptional mechanisms underlying myofibroblast phenotype would need to be fully elucidated, as well as the development of lineage tracers that are tissue/cell specific. Presently, there is no evidence demonstrating reversal of conjunctival fibrosis but the absence of evidence does not make it impossible. Prevention of ECM deposition is more likely to result in the restitution of conjunctival architecture. Therefore, it is important to identify patients who may be more susceptible to aberrant fibrosis at an early stage.

\section{Summary and perspective}

Conjunctival wound healing modulation continues to receive interest as it holds the key to GFS attaining and sustaining optimal IOP (10-14 mmHg) and preventing glaucoma progression associated with higher pressures. New treatments will arise from a better understanding of tissue specific associated molecular, cellular and biomechanical processes. Innovative solutions that can directly and indirectly modulate inflammatory and fibrotic processes at the optimal time, dosage and locality will result in more favourable outcomes. A combination of treatments or sequential treatments may be more efficacious than a single agent, particularly in individuals with a high risk of scarring. There are many areas for future investigation yet to be explored, in terms of novel or repurposed anti-fibrotic agents and sophisticated drug delivery systems. Genetic manipulation of cells involved in wound healing or in vivo reprogramming of myofibroblast may be future methods used to treat ocular fibrosis. Ultimately, the prize is great. Glaucoma is increasing exponentially with the rapidly ageing populations around the world, and a management plan that can cope with the scale of demand should involve surgical therapy as accessible and successful as cataract surgery.

We know from clinical evidence that pressures around the $10 \mathrm{mmHg}$ mark are associated with minimal optic nerve disease and minimal visual field progression over a decade. Micro-devices now allow a degree of surgical control and pressure control to achieve low pressures around $10 \mathrm{mmHg}$ safely and rapidly. This means the 101010 target we have proposed for glaucoma treatment $(10 \mathrm{~min}$ to conduct the treatment to achieve $10 \mathrm{mmHg}$ that lasts 10 years) would revolutionise the treatment of glaucoma and could become a possibility as long as we can control wound healing and fibrosis over the period. With the considerable advances that have been made in understanding and modulating fibrosis, this exciting prospect is in sight.

Acknowledgements The authors would like to acknowledge the National Institute for Health Research Biomedical Research Centre at Moorfields Eye Hospital NHS Foundation Trust and UCL Institute of Ophthalmology, Moorfields Eye Charity, the Michael and Ilse Katz Foundation, the Helen Hamlyn Trust, John Nolan and Fight for Sight (UK). PTK received the medal honouring Peter Watson at the XLIX Cambridge Ophthalmological Symposium in 2019.

\section{Compliance with ethical standards}

Conflict of interest PTK is a consultant to Aerie Pharmaceuticals, Glaukos, Novartis, Santen, Thea. PTK and SB are founders and shareholders of Optceutics Ltd. PTK is a founder, director and shareholder of Radiance Therapeutics.YB is a consultant to Optceutics Ltd. 
Publisher's note Springer Nature remains neutral with regard to jurisdictional claims in published maps and institutional affiliations.

\section{References}

1. Jayaram H, Jones MF, Eastlake K, Cottrill PB, Becker S, Wiseman J, et al. Transplantation of photoreceptors derived from human Muller glia restore rod function in the $\mathrm{P} 23 \mathrm{H}$ rat. Stem Cells Transl Med. 2014;3:323-33.

2. The Advanced Glaucoma Intervention Study (AGIS): 7. The relationship between control of intraocular pressure and visual field deterioration.The AGIS Investigators. Am J Ophthalmol. 2000;130:429-40.

3. Van Bergen T, Van de Velde S, Vandewalle E, Moons L, Stalmans I. Improving patient outcomes following glaucoma surgery: state of the art and future perspectives. Clin Ophthalmol. 2014;8:857-67.

4. Teller P, White TK. The Physiology of Wound Healing: Injury Through Maturation. Surg Clin N Am. 2009;89:599.

5. Luttikhuizen DT, Harmsen MC, Van Luyn MJA. Cellular and molecular dynamics in the foreign body reaction. Tissue Eng. 2006;12:1955-70.

6. Khaw PT, Chiang M, Shah P, Sii F, Lockwood A, Khalili A. Enhanced Trabeculectomy: The Moorfields Safer Surgery System. Dev Ophthalmol. 2017;59:15-35.

7. Saeedi OJ, Jefferys JL, Solus JF, Jampel HD, Quigley HA. Risk factors for adverse consequences of low intraocular pressure after trabeculectomy. J Glaucoma. 2014;23:e60-8.

8. Schlunck G, Meyer-ter-Vehn T, Klink T, Grehn F. Conjunctival fibrosis following filtering glaucoma surgery. Experimental Eye Research. 2016;142:76-82.

9. Wells AP, Crowston JG, Marks J, Kirwan JF, Smith G, Clarke JC, et al. A pilot study of a system for grading of drainage blebs after glaucoma surgery. J Glaucoma. 2004;13:454-60.

10. Bouremel Y, Lee RMH, Eames I, Brocchini S, Khaw PT. Novel approaches to model effects of subconjunctival blebs on flow pressure to improve clinical grading systems after glaucoma drainage surgery. PLoS ONE. 2019;14:e0221715.

11. Pakshir P, Hinz B. The big five in fibrosis: Macrophages, myofibroblasts, matrix, mechanics, and miscommunication. Matrix Biol. 2018;68-69:81-93.

12. WuDunn D. Intracameral urokinase for dissolution of fibrin or blood clots after glaucoma surgery. Am J Ophthalmol. 1997;124: 693-5.

13. Min J, Lukowski ZL, Levine MA, Meyers CA, Beattie AR, Schultz GS, et al. Prevention of ocular scarring post glaucoma filtration surgery using the inflammatory cell and platelet binding modulator saratin in a rabbit model. PLoS ONE. 2012;7:e35627.

14. Yuen D, Buys Y, Jin YP, Alasbali T, Smith M, Trope GE. Corticosteroids Versus NSAIDs on Intraocular Pressure and the Hypertensive Phase After Ahmed Glaucoma Valve Surgery. Journal of Glaucoma. 2011;20:439-44.

15. Yuki K, Shiba D, Kimura I, Ohtake Y, Tsubota K. Trabeculectomy With or Without Intraoperative Sub-Tenon Injection of Triamcinolone Acetonide in Treating Secondary Glaucoma. American Journal of Ophthalmology. 2009;147:1055-60.

16. Koval MS, Moster MR, Freidl KB, Waisbourd M, Jain SG, Ichhpujani P, et al. Intracameral Triamcinolone Acetonide in Glaucoma Surgery: A Prospective Randomized Controlled Trial. American Journal of Ophthalmology. 2014;158:395-401.

17. Vandervoort J, Ludwig A. Ocular drug delivery: nanomedicine applications. Nanomedicine. 2007;2:11-21.

18. Furino C, Boscia F, Cicinelli MV, Sborgia A, Alessio G. Subconjunctival sustained-release dexamethasone implant as an adjunct to trabeculectomy for primary open angle glaucoma. Indian Journal of Ophthalmology. 2016;64:251.

19. Hui A. Contact lenses for ophthalmic drug delivery. Clin Exp Optom. 2017;100:494-512.

20. Fakhraie G, Lopes JF, Spaeth GL, Almodin J, Ichhpujani P, Moster MR. Effects of postoperative cyclosporine ophthalmic emulsion $0.05 \%$ (Restasis) following glaucoma surgery. Clin Exp Ophthalmol. 2009;37:842-8.

21. Khaw PT, Sherwood MB, MacKay SL, Rossi MJ, Schultz G. Five-minute treatments with fluorouracil, floxuridine, and mitomycin have long-term effects on human Tenon's capsule fibroblasts. Arch Ophthalmol. 1992;110:1150-4.

22. Green E, Wilkins M, Bunce C, Wormald R. 5-Fluorouracil for glaucoma surgery. Cochrane Database Syst Rev 2014: CD001132.

23. Smith MF, Sherwood MB, Doyle JW, Khaw PT. Results of intraoperative 5-fluorouracil supplementation on trabeculectomy for open-angle glaucoma. Am J Ophthalmol. 1992;114: 737-41.

24. Lanigan L, Sturmer J, Baez KA, Hitchings RA, Khaw PT. Single intraoperative applications of 5-fluorouracil during filtration surgery: early results. Br J Ophthalmol. 1994;78:33-37.

25. Khaw PT, Doyle JW, Sherwood MB, Grierson I, Schultz G, McGorray S. Prolonged localized tissue effects from 5-minute exposures to fluorouracil and mitomycin C. Arch Ophthalmol. 1993;111:263-7.

26. Khaw PT, Sherwood MB, Doyle JW, Smith MF, Grierson I, McGorray $\mathrm{S}$, et al. Intraoperative and post operative treatment with 5-fluorouracil and mitomycin-c: long term effects in vivo on subconjunctival and scleral fibroblasts. Int Ophthalmol. 1992;16: $381-5$.

27. Cabourne E, Clarke JC, Schlottmann PG, Evans JR Mitomycin C versus 5-Fluorouracil for wound healing in glaucoma surgery. Cochrane Database Syst Rev 2015:CD006259.

28. Wells AP, Cordeiro MF, Bunce C, Khaw PT. Cystic bleb formation and related complications in limbus- versus fornix-based conjunctival flaps in pediatric and young adult trabeculectomy with mitomycin C. Ophthalmology. 2003;110:2192-7.

29. Constable PH, Crowston JG, Occleston NL, Khaw PT. The effects of single doses of beta radiation on the wound healing behaviour of human Tenon's capsule fibroblasts. $\mathrm{Br} \mathrm{J}$ Ophthalmol. 2004;88:169-73.

30. Khaw PT, Ward S, Grierson I, Rice NS. Effect of beta radiation on proliferating human Tenon's capsule fibroblasts. Br J Ophthalmol. 1991;75:580-3.

31. Kirwan JF, Constable PH, Murdoch IE, Khaw PT. Beta irradiation: new uses for an old treatment: a review. Eye (Lond). 2003;17:207-15.

32. Kirwan JF, Rennie C, Evans JR. Beta radiation for glaucoma surgery. Cochrane Database Syst Rev 2012:CD003433.

33. Dhalla K, Cousens S, Bowman R, Wood M, Murdoch I. Is Beta Radiation Better than 5 Flurouracil as an Adjunct for Trabeculectomy Surgery When Combined with Cataract Surgery? A Randomised Controlled Trial. PLoS ONE. 2016;11:e0161674.

34. El Mazar HM, Mandour SS, Mostafa MI, Elmorsy OA. Augmented Subscleral Trabeculectomy With Beta Radiation and Mitomycin C in Egyptian Glaucoma Patients. J Glaucoma. 2019; 28:637-42.

35. Cook CPA, Mustak H, Courtright P, Wetter J, du Toit N. Randomised clinical trial of trabeculectomy with mitomycin-C versus trabeculectomy with beta radiation. South African Ophthalmology Journal. 2018;13:11-14.

36. Perkins TW, Faha B, Ni M, Kiland JA, Poulsen GL, Antelman D, et al. Adenovirus-mediated gene therapy using human p21WAF1/Cip-1 to prevent wound healing in a rabbit model of glaucoma filtration surgery. Arch Ophthalmol. 2002;120:941-9. 
37. Lockwood A, Brocchini S, Khaw PT. New developments in the pharmacological modulation of wound healing after glaucoma filtration surgery. Curr Opin Pharmacol. 2013;13:65-71.

38. Hales AM, Chamberlain CG, McAvoy JW. Cataract induction in lenses cultured with transforming growth factor-beta. Invest Ophthalmol Vis Sci. 1995;36:1709-13.

39. Connor TB Jr, Roberts AB, Sporn MB, Danielpour D, Dart LL, Michels RG, et al. Correlation of fibrosis and transforming growth factor-beta type 2 levels in the eye. J Clin Invest. 1989;83:1661-6.

40. Lutty GA, Merges C, Threlkeld AB, Crone S, McLeod DS. Heterogeneity in localization of isoforms of TGF-beta in human retina, vitreous, and choroid. Invest Ophthalmol Vis Sci. 1993;34:477-87.

41. Picht G, Welge-Luessen U, Grehn F, Lutjen-Drecoll E. Transforming growth factor beta 2 levels in the aqueous humor in different types of glaucoma and the relation to filtering bleb development. Graefes Arch Clin Exp Ophthalmol. 2001;239: 199-207.

42. Mead AL, Wong TT, Cordeiro MF, Anderson IK, Khaw PT. Evaluation of anti-TGF-beta2 antibody as a new postoperative anti-scarring agent in glaucoma surgery. Invest Ophthalmol Vis Sci. 2003;44:3394-401.

43. Group CATTS, Khaw P, Grehn F, Hollo G, Overton B, Wilson R, et al. A phase III study of subconjunctival human anti-transforming growth factor beta(2) monoclonal antibody (CAT-152) to prevent scarring after first-time trabeculectomy. Ophthalmology. 2007;114:1822-30.

44. Akhurst RJ, Hata A. Targeting the TGFbeta signalling pathway in disease. Nat Rev Drug Discov. 2012;11:790-811.

45. Shu DY, Lovicu FJ. Myofibroblast transdifferentiation: The dark force in ocular wound healing and fibrosis. Prog Retin Eye Res. 2017;60:44-65.

46. Jinek M, Doudna JA. A three-dimensional view of the molecular machinery of RNA interference. Nature. 2009;457:405-12.

47. Hwang YH, Jung SA, Lyu J, Kim YY, Lee JH. Transforming Growth Factor-beta1-induced Human Subconjunctival Fibrosis is Mediated by MicroRNA 143/145 Expression. Invest Ophthalmol Vis Sci. 2019;60:2064-71.

48. Li N, Cui J, Duan X, Chen H, Fan F. Suppression of type I collagen expression by miR-29b via PI3K, Akt, and Sp1 pathway in human Tenon's fibroblasts. Invest Ophthalmol Vis Sci. 2012;53:1670-8.

49. Stewart AG, Thomas B, Koff J. TGF-beta: Master regulator of inflammation and fibrosis. Respirology. 2018;23:1096-7.

50. Meng XM, Nikolic-Paterson DJ, Lan HY. TGF-beta: the master regulator of fibrosis. Nat Rev Nephrol. 2016;12:325-38.

51. Hanna J, Hossain GS, Kocerha J. The Potential for microRNA Therapeutics and Clinical Research. Front Genet. 2019;10:478.

52. Wang Y, Wang J, Wei LJ, Zhu DM, Zhang JS. Biological function and mechanism of IncRNA-MEG3 in Tenon's capsule fibroblasts proliferation: By MEG3-Nrf2 protein interaction. Biomed Pharmacother. 2017;87:548-54.

53. Zhao Y, Zhang F, Pan Z, Luo H, Liu K, Duan X. LncRNA NR_003923 promotes cell proliferation, migration, fibrosis, and autophagy via the miR-760/miR-215-3p/IL22RA1 axis in human Tenon's capsule fibroblasts. Cell Death Dis. 2019;10:594.

54. Liu Y, Liu PP, Liu L, Zheng XS, Zheng H, Yang CC, et al. Triptolide inhibits TGF-beta-induced matrix contraction and fibronectin production mediated by human Tenon fibroblasts. Int $\mathbf{J}$ Ophthalmol. 2018;11:1108-13.

55. Gong W, Li J, Zhu G, Wang Y, Zheng G, Kan Q. Chlorogenic acid relieved oxidative stress injury in retinal ganglion cells through IncRNA-TUG1/Nrf2. Cell Cycle. 2019;18:1549-59.

56. Carmeliet P, Jain RK. Molecular mechanisms and clinical applications of angiogenesis. Nature. 2011;473:298-307.
57. Beddy D, Watson RW, Fitzpatrick JM, O'Connell PR. Increased vascular endothelial growth factor production in fibroblasts isolated from strictures in patients with Crohn's disease. Br J Surg. 2004;91:72-7.

58. Li Z, Van Bergen T, Van de Veire S, Van de Vel I, Moreau H, Dewerchin $\mathrm{M}$, et al. Inhibition of vascular endothelial growth factor reduces scar formation after glaucoma filtration surgery. Invest Ophthalmol Vis Sci. 2009;50:5217-25.

59. Cheng JW, Cheng SW, Wei RL, Lu GC. Anti-vascular endothelial growth factor for control of wound healing in glaucoma surgery. Cochrane Database Syst Rev 2016:CD009782.

60. Kahook MY. Bleb morphology and vascularity after trabeculectomy with intravitreal ranibizumab: a pilot study. Am J Ophthalmol. 2010;150:399-403e391.

61. Kahook MY, Schuman JS, Noecker RJ. Needle bleb revision of encapsulated filtering bleb with bevacizumab. Ophthalmic Surg Lasers Imaging. 2006;37:148-50.

62. Dong Z, Gong J, Liao R, Xu S. Effectiveness of multiple therapeutic strategies in neovascular glaucoma patients: A PRISMAcompliant network meta-analysis. Medicine (Baltimore). 2018;97: e9897.

63. Cornish KS, Ramamurthi S, Saidkasimova S, Ramaesh K. Intravitreal bevacizumab and augmented trabeculectomy for neovascular glaucoma in young diabetic patients. Eye (Lond). 2009;23: 979-81.

64. Akiyama H, Kachi S, Silva RL, Umeda N, Hackett SF, McCauley $\mathrm{D}$, et al. Intraocular injection of an aptamer that binds PDGF-B: a potential treatment for proliferative retinopathies. J Cell Physiol. 2006;207:407-12.

65. Lin X, Wen J, Liu R, Gao W, Qu B, Yu M. Nintedanib inhibits TGF-beta-induced myofibroblast transdifferentiation in human Tenon's fibroblasts. Mol Vis. 2018;24:789-800.

66. Conte E, Gili E, Fagone E, Fruciano M, Iemmolo M, Vancheri C. Effect of pirfenidone on proliferation, TGF-beta-induced myofibroblast differentiation and fibrogenic activity of primary human lung fibroblasts. Eur J Pharm Sci. 2014;58:13-9.

67. Tomasek JJ, Gabbiani G, Hinz B, Chaponnier C, Brown RA. Myofibroblasts and mechano-regulation of connective tissue remodelling. Nat Rev Mol Cell Biol. 2002;3:349-63.

68. Mori T, Kawara S, Shinozaki M, Hayashi N, Kakinuma T, Igarashi A, et al. Role and interaction of connective tissue growth factor with transforming growth factor-beta in persistent fibrosis: A mouse fibrosis model. J Cell Physiol. 1999;181:153-9.

69. Esson DW, Neelakantan A, Iyer SA, Blalock TD, Balasubramanian L, Grotendorst GR, et al. Expression of connective tissue growth factor after glaucoma filtration surgery in a rabbit model. Invest Ophthalmol Vis Sci. 2004;45:485-91.

70. Yamanaka O, Saika S, Ikeda K, Miyazaki K, Kitano A, Ohnishi Y. Connective tissue growth factor modulates extracellular matrix production in human subconjunctival fibroblasts and their proliferation and migration in vitro. Jpn J Ophthalmol. 2008;52:8-15.

71. Razzaque MS, Foster CS, Ahmed AR. Role of connective tissue growth factor in the pathogenesis of conjunctival scarring in ocular cicatricial pemphigoid. Invest Ophthalmol Vis Sci. 2003; 44:1998-2003.

72. Gooz M. ADAM-17: the enzyme that does it all. Crit Rev Biochem Mol Biol. 2010;45:146-69.

73. Wang Y, Tang Z, Xue R, Singh GK, Lv Y, Shi K, et al. TGFbeta1 promoted MMP-2 mediated wound healing of anterior cruciate ligament fibroblasts through NF-kappaB. Connect Tissue Res. 2011;52:218-25.

74. McCluskey P, Molteno A, Wakefield D, Di Girolamo N. Otago Glaucoma Surgery Outcome Study: the pattern of expression of MMPs and TIMPs in bleb capsules surrounding Molteno implants. Invest Ophthalmol Vis Sci. 2009;50:2161-4. 
75. Wong TT, Mead AL, Khaw PT. Matrix metalloproteinase inhibition modulates postoperative scarring after experimental glaucoma filtration surgery. Invest Ophthalmol Vis Sci. 2003;44:1097-103.

76. Mohamed-Ahmed AHA, Lockwood A, Li H, Bailly M, Khaw PT, Brocchini S. An Ilomastat-CD Eye Drop Formulation to Treat Ocular Scarring. Invest Ophthalmol Vis Sci. 2017;58:3425-31.

77. Sen E, Balikoglu-Yilmaz M, Bozdag-Pehlivan S, Sungu N, Aksakal FN, Altinok A, et al. Effect of doxycycline on postoperative scarring after trabeculectomy in an experimental rabbit model. J Ocul Pharmacol Ther. 2010;26:399-406.

78. Molnar J, Fong KS, He QP, Hayashi K, Kim Y, Fong SF, et al. Structural and functional diversity of lysyl oxidase and the LOXlike proteins. Biochim Biophys Acta. 2003;1647:220-4.

79. Park HY, Kim JH, Park CK. Lysyl oxidase-like 2 level and glaucoma surgical outcomes. Invest Ophthalmol Vis Sci. 2014;55:3337-43.

80. Van Bergen T, Marshall D, Van de Veire S, Vandewalle E, Moons L, Herman J, et al. The role of LOX and LOXL2 in scar formation after glaucoma surgery. Invest Ophthalmol Vis Sci. 2013;54:5788-96.

81. Tovell VE, Chau CY, Khaw PT, Bailly M. Rac1 inhibition prevents tissue contraction and MMP mediated matrix remodeling in the conjunctiva. Invest Ophthalmol Vis Sci. 2012;53:4682-91.

82. Honjo M, Tanihara H, Kameda T, Kawaji T, Yoshimura N, Araie M. Potential role of Rho-associated protein kinase inhibitor Y27632 in glaucoma filtration surgery. Invest Ophthalmol Vis Sci. 2007;48:5549-57.

83. Evelyn CR, Wade SM, Wang Q, Wu M, Iniguez-Lluhi JA, Merajver SD, et al. CCG-1423: a small-molecule inhibitor of RhoA transcriptional signaling. Mol Cancer Ther. 2007;6: 2249-60.

84. Tagalakis AD, Madaan S, Larsen SD, Neubig RR, Khaw PT, Rodrigues I, et al. In vitro and in vivo delivery of a sustained release nanocarrier-based formulation of an MRTF/SRF inhibitor in conjunctival fibrosis. J Nanobiotechnology. 2018;16:97.

85. Yu-Wai-Man C, Spencer-Dene B, Lee RMH, Hutchings K, Lisabeth EM, Treisman R, et al. Local delivery of novel MRTF/
SRF inhibitors prevents scar tissue formation in a preclinical model of fibrosis. Sci Rep. 2017;7:518.

86. Shao T, Li X, Ge J. Target drug delivery system as a new scarring modulation after glaucoma filtration surgery. Diagn Pathol. 2011;6:64.

87. Barton K, Budenz DL, Khaw PT, Tseng SC. Glaucoma filtration surgery using amniotic membrane transplantation. Invest Ophthalmol Vis Sci. 2001;42:1762-8.

88. Pakravan M, Rad SS, Yazdani S, Ghahari E, Yaseri M. Effect of early treatment with aqueous suppressants on Ahmed glaucoma valve implantation outcomes. Ophthalmology. 2014;121: 1693-8.

89. Law SK, Kornmann HL, Giaconi JA, Kwong A, Tran E, Caprioli J. Early Aqueous Suppressant Therapy on Hypertensive Phase Following Glaucoma Drainage Device Procedure: A Randomized Prospective Trial. J Glaucoma. 2016;25:248-57.

90. Liu Y, Ko JA, Yanai R, Kimura K, Chikama T, Sagara T, et al. Induction by latanoprost of collagen gel contraction mediated by human tenon fibroblasts: role of intracellular signaling molecules. Invest Ophthalmol Vis Sci. 2008;49:1429-36.

91. Jung KI, Woo JE, Park CK. Effects of aqueous suppressants and prostaglandin analogues on early wound healing after glaucoma implant surgery. Sci Rep. 2019;9:5251.

92. Than JYL, Al-Mugheiry TS, Gale J, Martin KR. Factors predicting the success of trabeculectomy bleb enhancement with needling. Br J Ophthalmol. 2018;102:1667-71.

93. Siriwardena D, Kotecha A, Minassian D, Dart JK, Khaw PT. Anterior chamber flare after trabeculectomy and after phacoemulsification. Br J Ophthalmol. 2000;84:1056-7.

94. Husain R, Liang S, Foster PJ, Gazzard G, Bunce C, Chew PT, et al. Cataract surgery after trabeculectomy: the effect on trabeculectomy function. Arch Ophthalmol. 2012;130:165-70.

95. Song G, Pacher M, Balakrishnan A, Yuan Q, Tsay HC, Yang D, et al. Direct Reprogramming of Hepatic Myofibroblasts into Hepatocytes In Vivo Attenuates Liver Fibrosis. Cell Stem Cell. 2016;18:797-808. 